evidence that chlorophyll is the source of one type of free electrons in an intact photosynthetic organism and that plastoquinone is the site of another type, the two types of signal have been correlated with the evolution of photosynthetic oxygen. Some particularly interesting studies of the photosynthetic rates of six races of Mimulus originating in diverse climates and altitudes indicated that climatic races within the same species may show differential patterns of response linked with variations in internal physiology.

The Department of Terrestrial Magnetism, in which the emphasis of the programme has markedly changed from earlier years, apart from studies of the intensity of the charged particles in the Van Allen trapped-radiation belt (as recorded during the transits of satellite Explorer VII through the belt between 1959 and 1960), was concerned with the application of physical techniques to a wide range of geophysical and biological problems. Using a simple and effective new procedure, it has been demonstrated that the deoxyribonucleic acid-like ribonucleic acid comprises about 1 per cent of the total ribonucleic acid of bacterial cells, and that it has a half-life during active synthesis of about $2 \mathrm{~min}$. If this ribonucleic acid is, in fact, the template for protein synthesis, a single mole. cule acts catalytically for the synthesis of many polypeptide chains. The method can also be used to exploit the specificity in the hybridization process, which depends on long regions of complementary nucleotide sequences in molecules of ribonucleic acid and deoxyribonucleic acid and it can also be used in studies of the transeription of genetic information and of differentiation.

With support of the National Science Foundation, the Department is establishing a new Carnegie Radio Astronomy Station in Argentina, and it is also constructing an interferometer array from parabolic disk antennæ to obtain precise positions of radio-noise sources in the sky. During the period covered by the report, the observational programme in radio astronomy continued and observations of the hydrogen gas content at the centre of our Galaxy confirmed that the motions of hydrogen close to the Galactic centre are complex and that the hydrogen gas is expanding as well as rotating. A major co-operative project of the Seismic Studies Group was an intensive study of the Earth's crust in Maine which demonstrated the very real limitations imposed on our ability to learn about crustal 'layers' by the customary methods of explosion seismology, and its findings postulate appreciably less granitic material than is customarily thought to be in a continental crust. The Group concerned with the radioactive dating of rocks participated in an exchange with the Geological and Mineralogical Institute of the University of Kyoto and has compiled a new map of agedistribution in erystalline basement rocks of North America. The Department is also associated with the work of the Committee on Image Tubes for Telescopes which has now reached a stage when a real gain in research work over the best photographic plates is just beginning to be realized by means of convenient (and permanent) scaled-off image tubes.

The Geophysical Laboratory focused much of its work in experimental petrology during the year on pyroxene minerals and the study of phase equilibria at higher pressures. Additional experimental data for constructing concepts of the Earth's mantle and crust are being obtained from an examination of the melting relations of silicates at high pressures. Much effort was expended on studies of the mineralogy of meteorites, and new investigations in organic geochemistry included analysis of Precambrian carbonaceous materials. Fatty acids were isolated from rocks as old as 500 million years, including myristic, palmitic and stearic acid, and the same acids have been isolated from recent sediments but with a marked difference in the relative proportions of acids. Much the longest of the departmental reports, that from the Geophysical Laboratory, includes summaries of more than fifty investigations.

At the Mount Wilson and Palomar Observatories an extensive study of the accumulated magnetograms to classify magnetic regions and correlate them with optical and radio phenomena showed that the unipolar magnetic regions of the Sun correlate in position with the calcium absorption phenomenon observed spectroscopically. Studies of the spectra of the major planets were commenced and two lines of the hydrogen molecule were found in the spectrum of Saturn. The analysis of high-dispersion spectra of Venus afforded evidence of large changes in the temperature of the atmosphere of Venus. Colourmagnitude diagrams have been constructed for many globular and galactic elusters and high-dispersion spectra used to make detailed quantitative chemical analyses of stellar atmospheres. New knowledge was obtained about differences in chemical composition among stars, the correlation of chemical composition and star movement, and fresh evidence on the expansion of the cosmos from studies of nearby galaxies by photoelectric scanning methods. From studies of the orbits around the nucleus of our Galaxy of a large number of dwarf stars, both normal and sub-dwarf types, the time of formation of the Galaxy out of the medium of the universe has been fixed at about 10 billion years ago.

The completion of the new building for the Department of Embryology was a feature of the year in which the Department's most striking progress was in continuation of its studies of the problems of muscle development. Investigation of the cytodifferentiation of embryonic skeletal muscle cells in dispersed cell culture using freshly isolated embryonic muscle cells showed a plating efficiency of about 10 per cent, and in plates cultivated for 10-13 days about 1 in 10 colonies showed unmistakable signs of skeletal muscle cell differentiation. Further tests showed that the medium is altered by the metabolic activity of cells cultured in it, and the results, impressive in themselves, represent something of a technical breakthrough in the task of cell culture. Other investigations include studies of the physiological aspect of frogembryo growth from the stage of the fertilized egg, of the nature of the testicular antigen in induced aspermatogenesis, of the role of deoxyribonuclease II during the metamorphosis of the tadpole, and a comprehensive study of the developing human eye, as well as a study of the vascular pattern of the human uterus with placenta in situ.

\title{
VASCULAR AND LYMPHATIC RESPONSES IN INJURY
}

SYMPOSIUM on the "Vaseular and Lymphatic
Responses in Injury", organized by the School of
Pathology of the newly established Faculty of Medicine
of the University of New South Wales, attracted pathol.
ogists from all parts of Australia, and was honoured by
the presence of Sir Roy Cameron, of University College
Hospital, London. The symposium was sponsored by Geigy Pharmaceuticals.

The first session on November 2 was devoted to problems of vascular structure and behaviour. The opening paper was given by Dr. A. A. Palmer (Sydney). Dr. Palmer pointed out that blood in capillaries is separated 
into a central zone containing corpuscles, and a peripheral zone composed of plasma. This 'plasma skimming' is increased by the addition of substances which increase the sedimentation rate of the blood, for example, high molecular weight polymers like dextran.

Next, Dr. W. E. Stehbens (Canberra) discussed the structure of vascular endothelium with particular reference to the existence of intercellular cement substance. Carbon, chylomicra and platelets were injected into the circulation but did not stick to the space between vascular endothelial cells on electron microscopic examination. Transparent glutinous masses present in the rabbit's ear chamber, and in the frog's web after the intravascular administration of carbon, were shown to be agglutinated masses of platelets with some fibrin, rather than cement substance. Accordingly, the evidence for the existence of capillary cement substance is inconclusive.

Dr. I. K. Buckley (Melbourne) described the structure of cellular and vascular reactions after injury. Injury was produced by a high-frequency electric current to ear chamber tissue. A progressively enlarging area showed vascular damage, and nearby granulocytes migrated towards this centre of injury. Thin-walled transparent globular structures, probably myelin figures, developed at the surface of heat-injured cells.

Dr. W. J. Cliff (Perth) described electron microscopic studies of the process of wound healing in the rabbit's ear chamber. Cell division occurred near, but not at, the tip of capillary sprouts. The tip of the cord consisted of a mass of endothelial cells, which gradually separated; the lumen was constituted by the intercellular space so formed. Basement membrane was probably produced by interaction between endothelium and the extravascular tissues, while both adventitial cells and smooth muscle cells probably developed from fibroblasts.

The session concluded with a report on the reaction of cells of peripheral and intermediate lymph to foreign materials by Dr. J. G. Hall (Canberra), read in his absence by Dr. B. Morris. Foreign materials were injected into the hind limb of a sheep the popliteal lymph node of which had been cannulated on either the afferent or efferent side. Radioactive isotope studies showed that a non. particulate antigen-human globulin-was not retained in the lymph node, whereas a particulate antigen, the avian red cell, was. About $50 \mathrm{~h}$ after the initial injection the cell population of the efferent lymph from the node began to change: initially, large immature cells poured into the lymph, followed later by lymphocytes and plasma cells. The output of RNA correlated quite closely with the plasma cell response.

The second session was given over mainly to the phenomena associated with inflammation. The session was opened by Sir Roy Cameron (London), who described the vascular and cellular events following injury.

The early reactions to injury are chemically mediated, mainly by histamine, aided in some species by 5-hydroxytryptamine. The beginning of increased permeability is delayed by tiny doses of antihistamine. The delayed vascular response of sustained vasodilatation and increased vascular permeability is more complex. These reactions may be promoted by plasma factors-kinins and permeability increasing globulins, and antagonized by vasoconstrictor amines; there may be augmented hist. amine synthesis to compensate for the explosive loss of histamine which occurs immediately after injury. This may all be triggered by a protein which is readily susceptible to denaturation; when denatured, it is postulated that it no longer acts as an enzyme inactivator. The eycle is then started.

The next paper was a discussion of the effect of injury on the permeability of the vascular endothelium by Prof. F. C. Courtice (Canberra). Rabbits were studied in which excessively high plasma levels of cholesterol and triglycerides respectively had been induced. It was found that the higher the ratio of protein to lipid and the more dense the fraction the smaller the lipoprotein particle. The smaller molecules passed across the membrane more readily than the larger. After heat injury in hyperlipæmia, there was an increase in the lipids in the lymph, which may become milky. It is postulated that materials pass through the capillary wall in vesicles or otherwise and that the polysaccharides in the basement membrane act as a sieve. After injury, in hyperlipæmic animals, lipoproteins also passed across the endothelium in arteries, and were deposited below the internal elastic lamina.

Vascular permeability changes in ultra-violet injury were described by Dr. G. Logan and Prof. D. L. Wilhelm (Sydney), using the technique of 'blueing'. In ultra. violet, as in other types of injury, the inflammatory reaction is characterized by three vascular responsesvasodilatation, increased permeability and migration of leucocytes. In this case, however, they develop relatively slowly and can be dissected apart. Erythema and increased permeability each occur in two phases: the leucocytosis rises and falls 3-4 $\mathrm{h}$ in front of the late phase of increased permeability. The early phase of increased permeability is mediated by histamine in guinea pigs and rabbits, by 5 -hydroxytryptamine in rats. The mediator of the late phase is unknown.

Dr. J. Margolis (Sydney) gave an account of his quantitative work on plasma kinins. These factors may be released from plasma by kallikrein, trypsin or the glandular 'kallikreins' from saliva and urine. The normal release of these factors is dependent on three factors: component $A$, or kallikreininogen; Hageman factor, which is also involved in blood clotting; and compound $B$. a protein easily adsorbed on to glass. Hageman factor is easily activated by contact with a negatively charged surface. Trypsin and the glandular kallikreins are independent of the Hageman factor.

The plasma kinins are bradykinin, a nonapeptide, and kallidin, a decapeptide. These may be loosely attached to plasma globulins as kininogens; plasma kallikrein may act on one substrate (globulin plus nonapeptide) corresponding to the nonapeptide precursor compound $B$. The others act on both substrates (globulin plus nonapeptide, and globulin plus decapeptide); trypsin produces bradykinin, and glandular kallikrein produces both the nona- and deca-peptides. The decapeptides are attacked by a plasma peptidase to produce a nonapeptide which is probably bradykinin.

The last paper of the day was given by Dr. B. Morris (Canberra). He described techniques for producing permanent lymphatic fistulæ in sheep. The blood vascular and lymphatic flow in an area are both altered by exercise. Thus, exercise induces a cessation of flow of intestinal lymph and a fall in hepatic lymph flow, but an increase in the flow of leg lymph. In the exercised lactating sheep mammary flow increases. Exercise also increases the rate of turnover of radioactive protein between the intra. vascular and extravascular compartments.

The main topic of the third session (November 3) was the response of cells in inflammation. Dr. J. V. Hurley (Melbourne) demonstrated the existence of an active principle which induces leucocytic migration. This substance may be derived from burn extracts, from polymorphs and from activated serum; it is non-dialysable, is destroyed by trypsin, but not by ethylenediamine tetraacetic acid or any of the permeability inhibitors.

Next, the question of how cells recognize one another and how cell behaviour is controlled by other cells was explored by Dr. S. V. Boyden (Canberra). The chemotactic response, the first part of the recognition of foreign material, was investigated. Cells were observed while migrating through a millipore collodion membrane towards foreign material on the other side; the more foreign the material, the greater the tendency for the phagocyte to migrate towards it. In the case of cellulose particles, cell migration was much more marked in the 
presence of serum. It was concluded that the power of recognition of the foreign material lay not in the cells but in the serum.

Dr. V. J. McGovern (Sydney) reported on work carried out in collaboration with Dr. D. Bloomfield. The ability of leucocytes to stick to vascular endothelium was investigated using en face preparations. Saline was injected into the adventitia of arteries, and 1.5-3 h later leucocytes stuck to the uninjured endothelial surface. These penetrated the endothelium and continued to do so until about $18 \mathrm{~h}$ after the original trauma. If in making the injection the endothelium was injured to the point of thrombosis, or nearly so, leucocytes stuck to the endothelium much more rapidly. The worse the injury, the sooner did they stick.

The structure of macrophages was the subject of an account by Dr. I. Carr (Sydney). The common feature in granulomata induced by introduction of foreign materials was the presence of spherical masses of phagocytic cells arranged around the foreign material. It was suggested that lipid was phagocytosed in these circum stances by a process which involved the membrane disappearing and the materia] passing into the cell free of membrane. The hypothesis was put forward that the cell membrane may dissolve in the extracellular lipid. The giant cells in these lesions probably are formed largely by cell fusion.

The last paper was that of Dr. E. G. Cleary (Sydney). Dr. Cleary described his analysis of the content of collagen and elastin in samples of human arterial intima and media. More elastin was present proximally and more collagen distally, but the sum of these was constant. The elastin content was probably increased in the new-born; collagen decreased with age. Even muscular arteries were found to contain as much as 52 per cent collagen by chemical analysis.

In his summing up, Sir Roy Cameron commented on the wide range of interest of the symposium, which indicated the emergence of a distinctive Australian approach to experimental pathology. I. CARR

\section{GELATINE AND GLUE RESEARCH}

\begin{abstract}
THE twenty-fourth research panel meeting of the British Gelatine and Glue Research Association was held at the Strand Palace Hotel, London, W.C.2, on November 7. The chairman of the Association, Mr. S. G. Hudson (Richard Hodgson and Sons, Bevérley), presided and directed attention to the place of the first paper on wool structure in the research panel series, which has lately included papers on other complex proteins such as collagen ${ }^{1}$ and elastin ${ }^{2}$.

Dr. A. Robson (Wool Industries Research Association, 'Torridon', Leeds) contributed a paper on 'The Sulphur Balance in Wool". In the past it has often been noted that total sulphur analyses for wool are significantly larger than the sum of the individual sulphur-containing compounds estimated by other methods. Most of the sulphur in wool is in cystine, and colorimetric and chromatographic assays of cystine in wool hydrolysates differ by 10 per cent. This fact caused the problem of the sulphur balance to be re-examined. Hydrolysates of wool labelled with sulphur-35 were chromatographed and found to contain several unidentified sulphur compounds, of which one became very abundant when hydrolysis was prolonged. It was identified as bis ( $\beta$-amino- $\beta$-carboxyethyl) trisulphide, that is, the trisulphide analogue of cystine $^{3}$. Thus some of the cystine decomposes during wool hydrolysis and from careful analyses it is concluded that wool contains sulphur only in the form of cystine, cysteine and methionine, thus bringing it into line with other proteins and removing the discrepancy in the sulphur balance. The discussion centred around the mechanism by which the trisulphide artefact might be formed.
\end{abstract}

In the second paper, Mr. L. J. H. Slack (English Abrasives Corporation, Ltd., Tottenham, London, N.17) discussed "Comparisons of Natural and Synthetic Glues in the Coated Abrasives Industry". The industry is a key one in relation to many others of much greater size, for example, metal working. There is a considerable variety of coated abrasives and the glues used include hide glue and synthetics based on alkyds, urea, melamine and phenols. The merits and demerits of hide glue were discussed, and it was concluded that there was need for improvement of the final film properties to enable the natural material to maintain its competitive position.

The director of research, Dr. D. A. Sutton, reported on "Some Recent Impressions of the American Gelatine and Glue Industry". "Emphasis was laid on the speed and hygienic control which are characteristic of the production of American pigskin gelatine; these factors are reflected in the quality of the final product. The plants and processes used were briefly outlined and the other types of gelatine produced were commented on. Attention was directed to the extent to which hide and bone glue production are mechanized in the United States and this factor enables natural glue to be sold at a low price in spite of very high labour rates. There is no central research organization parallel to the British Gelatine and Glue Research Association, but many large companies have large well-equipped laboratories covering all their products and including facilities for gelatine and glue.

$$
\text { D. A. Sutton }
$$

${ }^{1}$ Sutton, D. A., Nature, 196, 223 (1962).

2 Sutton, D. A., Nature, 196, 422 (1962).

${ }^{3}$ Fletcher, J. C., and Robson, A., Nature, 195, 1308 (1962).

\section{THE BRITISH LEATHER MANUFACTURERS' RESEARCH ASSOCIATION}

\begin{abstract}
$A^{N}$ $\mathrm{N}$ outstanding feature of the past year was the strengthening of contacts between the British Leather Manufacturers' Research Association and the footwear industry. The great value of these contacts lies in showing the Research Association how it can adjust its research programme to help the leather industry to meet the requirements of its main customer and they ensure that the Association's work is relevant to the present needs of the footwear industry. The demands made on leather arising from the heat and steam lasting
\end{abstract}

of shoe uppers, for example, are being met and these new methods, which have great advantages, have been established and are likely to increase. A great deal of work has been done on the assessment of different leathers and finishes for these new treatments.

Investigations of skin proteins have continued at the Association's research laboratories, Milton Park, Egham, Surrey, but new emphasis is being given to seasonal variations in lambskins. The methods developed in this long-continued study of the constituents of skin have also 